\section{PREVALÊNCIA E FATORES ASSOCIADOS À REALIZAÇÃO DE MAMOGRAFIA E EXAME CITOPATOLÓGICO}

\author{
Prevalence and factors associated with the performance of \\ mammography and pap smear test \\ Prevalencia y factores asociados a la realización de mamografía \\ y examen histopatologico
}

Artigo Original

\section{RESUMO}

Objetivo: Estimar a prevalência e os fatores associados à realização de mamografia e exame citopatológico em mulheres da cidade de Maringá, Paraná. Métodos: Estudo transversal, de base populacional, feito com 345 mulheres com idade superior a 20 anos, no período de março de 2011 a abril de 2012. Realizou-se entrevista por meio de um questionário proposto pelo Ministério da Saúde, o qual abordava aspectos sociodemográficos, fatores de risco para doenças crônicas não transmissíveis e questões relacionadas ao rastreamento mamográfico e citopatológico. Os dados foram analisados mediante análise bivariada, análise bruta mediante Odds Ratio (OR) e qui-quadrado por meio do programa Epi Info 3.5.1, e análise multivariada por meio da regressão logística, realizada com o programa Statistica 7.1, com nível de significância de 5\% e intervalo de confiança de $95 \%$. Resultados: A média de idade das mulheres foi de 52,19 $( \pm 5,27)$ anos. A maioria $(56,5 \%)$ apresentou de 0 a 8 anos de estudo. Além disso, 84,6\% $(\mathrm{n}=266)$ das mulheres realizaram o exame de Papanicolau e $74.3 \%(n=169)$, a mamografia. Foram associadas à menor realização de Papanicolau as mulheres com escolaridade entre 9 e 11 anos de estudo $(\mathrm{p}=0,01)$, e quanto à mamografia, tiveram menor adesão as mulheres sem plano de saúde privado $(p<0,01)$. Conclusão: $A$ cobertura da mamografia e do Papanicolau foi satisfatória entre as mulheres da cidade de Maringá, Paraná. A baixa escolaridade e as mulheres que dependiam da rede pública de saúde tiveram menor adesão à realização da mamografia.

Descritores: Fatores de Risco; Neoplasias da Mama; Neoplasias Uterinas; Mamografia.

\section{ABSTRACT}

Objective: To estimate the prevalence and factors associated with the performance of mammography and pap smear test in women from the city of Maringá, Paraná. Methods: Population-based cross-sectional study conducted with 345 women aged over 20 years in the period from March 2011 to April 2012. An interview was carried out using a questionnaire proposed by the Ministry of Health, which addressed sociodemographic characteristics, risk factors for chronic noncommunicable diseases and issues related to mammographic and pap screening. Data were analyzed using bivariate analysis, crude analysis with odds ratio (OR) and chi-squared test using Epi Info 3.5.1 program; multivariate analysis using logistic regression was performed using the software Statistica 7.1, with a significance level of 5\% and a confidence interval of 95\%. Results: The mean age of the women was $52.19( \pm 5.27)$ years. The majority (56.5\%) had from 0 to 8 years of education. Additionally, $84.6 \%(n=266)$ of the women underwent pap smear and $74.3 \%(n=169)$ underwent mammography. The lower performance of pap smear test was associated with women with 9-11 years of education $(p=0.01)$, and the lower performance of mammography was associated with women without private health insurance $(p<0.01)$. Conclusion: The coverage of mammography and pap smear test was satisfactory among the women from Maringá, Paraná. Low education level and women who depended on the public health system presented lower performance of mammography.

Descriptors: Risk Factors; Breast Neoplasms; Uterine Neoplasms; Mammography.
Tiara Cristina Romeiro Lopes ${ }^{(1)}$ Angela Andréia França Gravena $^{(1)}$

Cátia Millene Dell Agnolo ${ }^{(2)}$ Sheila Cristina RochaBrischiliari $^{(1)}$

Marcela de Oliveira Demitto ${ }^{(1)}$ Maria Dalva de Barros Carvalho $^{(1)}$ Sandra Marisa Pelloso ${ }^{(1)}$

1) Universidade Estadual de Maringá UEM - Maringá (PR) - Brasil

2) Hospital Universitário Regional de Maringá - HURM - Maringá (PR) - Brasil
Recebido em: 22/04/2015 Revisado em: 10/07/2015 Aceito em: 26/08/2015 


\section{RESUMEN}

Objetivo: Estimar la prevalencia y los factores asociados a la realización de mamografía y examen histopatologico de mujeres de la ciudad de Maringá, Paraná. Métodos: Estudio transversal, de base poblacional con 345 mujeres de edad superior a 20 años en el periodo entre marzo de 2011 y abril de 2012. Se realizó una entrevista a través de un cuestionario propuesto por el Ministerio de la Salud el cual incluye aspectos sociodemográficos, factores de riesgo para las enfermedades crónicas no transmisibles y cuestiones relacionadas al rastreo mamográfico y histopatologico. Los datos fueron analizados a través del análisis bivariado, análisis bruto con el Odds Ratio (OR) y el chi-cuadrado a través del programa Epi Info 3.5.1, y análisis multivariado a través de la regresión logística realizada con el programa Stadistica 7.1, y nivel de significancia del 5\% e intervalo de confianza del 95\%. Resultados: La media de edad de las mujeres fue de $52,19( \pm 5,27)$ años. La mayoría $(56,5 \%)$ tenía entre 0 y 8 años de estudio. Además, el 84,6\% $(n=266)$ de las mujeres realizaron el Papanicolau y el 74,3\% (n=169) la mamografia. Las mujeres que tenían entre 9 y 11 años de estudio estuvieron asociadas con menos realización del Papanicolau $(p=0,01)$ y respecto la mamografia, las mujeres sin seguro de salud privado tuvieron menos adhesión al examen $(p<0,01)$. Conclusión: La cobertura de la mamografia y del Papanicolau fue satisfactoria en las mujeres de la ciudad de Maringá, Paraná. La baja escolaridad y las mujeres dependientes de la red pública de salud tuvieron menos adhesión en la realización de la mamografía.

Descriptores: Factores de Riesgo; Neoplasias de la Mama; Neoplasias Uterinas; Mamografía.

\section{INTRODUÇÃO}

Dados do Instituto Nacional de Câncer (INCA) apontam que o câncer de mama é o que mais atinge a população feminina no mundo inteiro, tanto em países em desenvolvimento quanto em países desenvolvidos. O câncer de colo de útero, por sua vez, é o mais incidente em países menos desenvolvidos. No Brasil, as estimativas para o ano de 2014, válidas também para 2015, são de 57.120 e 15.590 casos novos de câncer mamário e colo do útero, respectivamente ${ }^{(1)}$.

Dados da Agência Internacional de Pesquisas com Câncer (IARC) apontam que, em 2012, o câncer de mama teve uma incidência mundial de 1.676 .633 casos, e o número de mortes foi de 521.817 . O câncer do colo uterino apresentou, no mesmo ano, 527.624 casos e um número estimado de óbitos de 265.653 mil mulheres ${ }^{(2)}$.

Ações de promoção da saúde são de fundamental importância na detecção precoce dos cânceres de mama e útero, sendo passíveis de cura, dependendo do estágio em que são identificados. Dentre as práticas recomendadas para o rastreamento do câncer de mama, encontra-se o exame radiológico das mamas (mamografia), considerado o padrão ouro para o rastreamento da população de risco ${ }^{(3)}$. No que se refere ao câncer de colo uterino, o exame de Papanicolau, também conhecido por citologia oncótica, Paptest, dentre outros, é considerado a principal estratégia para sua detecção e prevenção $0^{(4)}$.

De acordo com o Ministério da Saúde, deve-se realizar o exame clínico anual das mamas a partir dos 40 anos, e o exame de mamografia a cada dois anos para mulheres de 50 a 69 anos. Porém, para mulheres pertencentes a grupos populacionais com risco elevado de desenvolver o câncer de mama (com história familiar de câncer da mama em parentes de primeiro grau antes dos 50 anos de idade; história familiar de câncer da mama bilateral ou de ovário em parentes de primeiro grau em qualquer idade; história familiar de câncer da mama masculina; ou mulheres com diagnóstico histopatológico de lesão mamária proliferativa com atipia ou neoplasia lobular in situ), o exame clínico da mama e a mamografia devem ser realizados a partir de 35 anos, anualmente ${ }^{(5)}$.

Para o rastreamento de câncer do colo do útero, o Ministério da Saúde adota como norma a recomendação da Organização Mundial da Saúde (OMS), que propõe a realização do exame citopatológico do colo do útero em mulheres de 25 a 60 anos de idade uma vez ao ano e, após dois exames anuais consecutivos negativos, a cada três $\operatorname{anos}^{(5)}$.

Após exploração da literatura, percebe-se que fatores associados à realização da mamografia e exame citopatológico são ainda pouco conhecidos no Brasil, e só a partir desse conhecimento prévio será possível traçar estratégias para trabalhar a promoção da saúde nas mulheres. Assim delineou-se a presente pesquisa, que teve como objetivo estimar a prevalência e os fatores associados à realização de mamografia e exame citopatológico em mulheres da cidade de Maringá, Paraná.

\section{MÉTODOS}

Os dados utilizados no presente estudo são provenientes da pesquisa "Monitoramento de fatores de risco para doenças crônicas não transmissíveis em adultos da cidade de Maringá, Paraná", realizada pelo grupo de pesquisa em saúde da mulher, no período de março de 2011 a abril de 2012. Trata-se de um estudo transversal de base populacional, por meio de inquérito domiciliar, para monitorar os fatores de risco e proteção para doenças crônicas não transmissíveis na população com idade $\geq 18$ anos.

Estimou-se o tamanho da amostra com o objetivo de assegurar representatividade para o evento em estudo. Calculou-se a amostra utilizando as informações do censo 
demográfico referente à população adulta (idade $\geq 18$ anos), relatadas pelo Instituto Brasileiro de Geografia e Estatística $(\mathrm{IBGE})^{(6)}$, no ano de 2010, para o município de Maringá, perfazendo um total de 273.674 adultos. Estabeleceu-se o número de 460 indivíduos a serem estudados. Esse número permitiu estimar, com coeficiente de confiança de $95 \%$ e erro máximo de cerca de cinco pontos percentuais, a frequência de qualquer fator de risco na população estudada e $20 \%$ de fator de confusão ${ }^{(7)}$.

A seleção da amostra teve como unidades de referência as áreas de expansão demográfica (AEDs) segundo o IBGE, totalizando em Maringá 21 AEDs. De cada setor foi selecionada uma amostra aleatória simples, proporcional ao número de adultos residentes em cada um desses setores, tendo em vista o tamanho da amostra (460). Devido à quantidade de indivíduos a ser pesquisada em cada setor ser proporcional ao tamanho deste, e para proporcionar melhor distribuição para efeito de vizinhança, foi sorteado um domicílio e saltado três. Não coincidindo a casa escolhida com a presença do adulto, foi procurado o domicílio seguinte, reiniciando-se o processo em cada entrevista. $\mathrm{Na}$ existência de mais de um indivíduo no domicílio, foi realizado sorteio entre eles.

Incluíram-se 345 mulheres com 20 anos ou mais. Para análise do rastreamento referente à realização de Papanicolau, foram utilizados os dados de mulheres com 20 anos ou mais (345). Para o rastreamento de mamografia, foram utilizados os dados respectivos das mulheres com idade superior a 40 anos $(268)^{(8)}$.

Aplicou-se o questionário do sistema de Vigilância de Fatores de Risco e Proteção para Doenças Crônicas por Inquérito Telefônico (VIGITEL), cuja implantação pelo Ministério da Saúde se deu em 2006 nos 26 estados brasileiros e no Distrito Federal, a fim de monitorar a frequência e distribuição dos principais determinantes das doenças crônicas não transmissíveis (DCNTs) e seus fatores de risco $^{(9)}$.

As variáveis abordadas foram: idade; cor (branca, negra, parda, amarela); estado civil (casada, solteira, divorciada/separada/viúva); escolaridade (0-8; 9-11 e 12 anos de estudo ou mais); ocupação remunerada (sim ou não); tabagismo (fumante, não fumante e ex-fumante); atividade física insuficiente no lazer, definida por ausência no lazer de atividade física leve ou moderada por pelo menos $30 \mathrm{~min} / \mathrm{dia}$ em cinco ou mais dias por semana ou de atividades de intensidade vigorosa por pelo menos $20 \mathrm{~min} / \mathrm{dia}$ em três ou mais dias da semana ${ }^{(10)}$; consumo abusivo de bebida alcoólica (mais que quatro doses em uma única ocasião, pelo menos uma vez nos últimos 30 dias uma dose de bebida alcoólica corresponde a uma lata de cerveja, uma taça de vinho ou uma dose destilada); estado nutricional, analisado pelo índice de massa corporal (IMC) e classificado de acordo com os critérios estabelecidos pela Organização Mundial da Saúde ${ }^{(11)} \mathrm{em}$ peso adequado $\left(\mathrm{IMC}<25 \mathrm{~kg} / \mathrm{m}^{2}\right)$ e excesso de peso $\left(\geq 25 \mathrm{~kg} / \mathrm{m}^{2}\right)$ no qual peso e altura foram autorreferidos; assim como saúde de modo geral (muito bom, bom, regular, ruim/muito ruim); plano de saúde privado (sim ou não). A realização do exame de mamografia e citopatológico alguma vez na vida foram questionadas, bem como o tempo (menos de 1 ano; entre 1 e 2 anos; entre 2 e 3 anos, entre 3 e 5 anos; 5 ou mais anos e não lembra).

Para análise bivariada do exame de Papanicolau e mamografia com as variáveis independentes, foi utilizada a análise bruta mediante Odds Ratio (OR), e qui-quadrado por meio do programa Epi Info 3.5.1. Na etapa seguinte, foram selecionadas as variáveis cujo valor do nível descritivo de significância do teste foi menor que 0,20 , utilizando a análise multivariada, por meio da regressão logística, estudando as variáveis em relação à realização do exame de Papanicolau e mamografia, realizadas com o programa Statistica 7.1, com nível de significância de 5\% e intervalo de confiança de $95 \%$.

O estudo foi aprovado pelo Comitê Permanente de Ética em Pesquisa com Seres Humanos da Universidade Estadual de Maringá, com o parecer nº. 30564/2012.

\section{RESULTADOS}

$\mathrm{Na}$ amostra elegível para esta pesquisa, foram avaliadas 345 mulheres com idade superior a 20 anos. A média de idade foi de $52,19( \pm 5,27)$ anos. Com relação à escolaridade, a maioria apresentou de 0 a 8 anos de estudo $(\mathrm{n}=195,56,55 \%)$.

Cerca de 67,8\% ( $\mathrm{n}=234)$ eram casadas/unidas e $65,2 \%(\mathrm{n}=225)$, de cor branca. Grande parte não praticava atividade física $(n=301 ; 87,2 \%), 7,5 \%(n=26)$ e $8,4 \%(n=29)$ relataram consumo abusivo de álcool e eram fumantes, respectivamente. Quanto ao estado nutricional, 54,9\% $(\mathrm{n}=147)$ apresentaram excesso de peso. A saúde, de modo geral, foi considerada ruim e muito ruim por $7,2 \%(n=25)$, e $57,1 \%(\mathrm{n}=197)$ relataram possuir plano de saúde privado (Tabela I).

Com relação às práticas preventivas de Papanicolau e mamografia, observou-se que $91,3 \%(n=315)$ das mulheres com idade superior a 20 anos realizaram alguma vez o exame de Papanicolau, e destas, 84,6\% $(\mathrm{n}=266)$ o fizeram nos últimos três anos. Quanto à mamografia, $85,1 \%(\mathrm{n}=228)$ das mulheres com idade superior aos 40 anos a realizaram, sendo 74,3\% $(\mathrm{n}=169)$ nos últimos dois anos.

Nas Tabelas II e III, encontram-se as prevalências de realização dos exames conforme características 
Tabela I - Distribuição percentual da população feminina segundo características sociodemográficas e comportamentos relacionados à saúde. Maringá-PR, 2012.

\begin{tabular}{|c|c|c|}
\hline Variáveis e categorias & $\mathbf{n}$ & $\%$ \\
\hline \multicolumn{3}{|l|}{ Idade (anos) } \\
\hline 20-39 & 77 & 22,3 \\
\hline $40-59$ & 145 & 42,0 \\
\hline$\geq 60$ & 123 & 35,7 \\
\hline \multicolumn{3}{|l|}{ Escolaridade (anos) } \\
\hline $0-8$ & 195 & 56,5 \\
\hline $9-11$ & 95 & 27,5 \\
\hline 12 ou mais & 55 & 15,9 \\
\hline \multicolumn{3}{|l|}{ Estado Civil } \\
\hline Solteira & 39 & 11,3 \\
\hline Casada/Unida & 234 & 67,8 \\
\hline Divorciada/Separada/Viúva & 72 & 20,9 \\
\hline \multicolumn{3}{|l|}{ Cor } \\
\hline Branca & 225 & 65,2 \\
\hline Negra & 9 & 2,6 \\
\hline Parda & 95 & 27,5 \\
\hline Amarela & 15 & 4,3 \\
\hline \multicolumn{3}{|l|}{ Ocupação remunerada } \\
\hline Sim & 108 & 31,3 \\
\hline Não & 234 & 67,8 \\
\hline \multicolumn{3}{|l|}{ Atividade física no lazer } \\
\hline Ativo & 44 & 12,8 \\
\hline Inativo & 301 & 87,2 \\
\hline \multicolumn{3}{|l|}{ Consumo abusivo de álcool } \\
\hline Sim & 26 & 7,5 \\
\hline Não & 319 & 92,5 \\
\hline \multicolumn{3}{|l|}{ Tabagismo } \\
\hline Fumante & 29 & 8,4 \\
\hline Não fumante & 259 & 75,1 \\
\hline Ex-fumante & 57 & 16,5 \\
\hline \multicolumn{3}{|l|}{ IMC* $\left(\mathrm{kg} / \mathrm{m}^{2}\right)$} \\
\hline$<25$ & 121 & 45,1 \\
\hline$\geq 25$ & 147 & 54,9 \\
\hline \multicolumn{3}{|l|}{ Saúde de modo geral } \\
\hline Muito bom & 50 & 14,5 \\
\hline Bom & 165 & 47,8 \\
\hline Regular & 105 & 30,4 \\
\hline Ruim / muito ruim & 25 & 7,2 \\
\hline \multicolumn{3}{|l|}{ Plano de Saúde } \\
\hline Sim & 197 & 57,1 \\
\hline Não & 148 & 42,9 \\
\hline
\end{tabular}

*índice de massa corporal; $\mathrm{n}=268$ mulheres avaliadas.

sociodemográficas e comportamentos de saúde. Encontrouse menor prevalência de realização do exame de Papanicolau em mulheres solteiras $(p<0,01)$ e divorciada/separada/viúva $(\mathrm{p}=0,01)$ (Tabela II).

Quanto ao exame de mamografia, este foi realizado de modo inferior nas mulheres solteiras $(\mathrm{p}<0,01)$ e naquelas sem plano de saúde privado $(\mathrm{p}<0,01)$ (Tabela III).
Após análise por regressão logística, foi associada com a realização do exame citopatológico a variável escolaridade, na qual mulheres com escolaridade de 9 a 11 anos apresentaram menos chances de realizarem esse exame (Tabela IV). Com relação ao exame de mamografia, o fato de a mulher não possuir plano de saúde privado reduziu as chances de realização (Tabela V). 
Tabela II - Realização de exame de Papanicolau segundo características sociodemográficas e comportamentos relacionados à saúde. Maringá-PR, 2012.

\begin{tabular}{|c|c|c|c|}
\hline Variáveis & $\begin{array}{c}\text { Realização do exame } \\
\text { Papanicolau } \\
\text { n (\%) } \\
\end{array}$ & $\begin{array}{c}\text { OR } \\
(\mathrm{IC} 95 \%)\end{array}$ & p valor \\
\hline \multicolumn{4}{|l|}{ Idade } \\
\hline $20-39$ & $70(90,9)$ & 1,0 & \\
\hline $40-59$ & $134(92,4)$ & $1,22(0,41-3,59)$ & 0,69 \\
\hline$\geq 60$ & $111(90,2)$ & $0,93(0,31-2,68)$ & 0,87 \\
\hline \multicolumn{4}{|l|}{ Escolaridade (anos) } \\
\hline $0-8$ & $177(90,8)$ & $1,43(0,51-3,91)$ & 0,44 \\
\hline $9-11$ & $90(94,7)$ & $2,63(0,70-10,17)$ & 0,12 \\
\hline 12 ou mais & $48(87,3)$ & 1,0 & \\
\hline \multicolumn{4}{|l|}{ Estado Civil } \\
\hline Casada & $223(95,3)$ & 1,0 & \\
\hline Solteira & $30(76,9)$ & $0,16(0,06-0,47)$ & $<0,01$ \\
\hline Divorciada/Separada/Viúva & $62(86,1)$ & $0,31(0,11-0,82)$ & 0,01 \\
\hline \multicolumn{4}{|l|}{ Cor } \\
\hline Branca & $210(92,9)$ & 1,0 & \\
\hline Negra & $9(100,0)$ & - & \\
\hline Parda & $83(87,4)$ & $0,53(0,22-1,25)$ & 1,10 \\
\hline Amarela & $13(86,7)$ & $0,50(0,09-3,48)$ & 0,31 \\
\hline \multicolumn{4}{|l|}{ Ocupação remunerada } \\
\hline $\operatorname{Sim}$ & $99(90,8)$ & 1,0 & \\
\hline Não & $216(91,5)$ & $1,09(0,46-2,56)$ & 0,87 \\
\hline \multicolumn{4}{|l|}{ Atividade física no lazer } \\
\hline Ativo & $40(90,9)$ & 1,0 & \\
\hline Inativo & $275(91,4)$ & $0,95(0,35-3,38)$ & 1,00 \\
\hline \multicolumn{4}{|l|}{ Consumo abusivo de álcool } \\
\hline $\operatorname{Sim}$ & $23(88,5)$ & $0,71(0,19-3,18)$ & 0,48 \\
\hline Não & $292(91,5)$ & 1,0 & \\
\hline \multicolumn{4}{|l|}{ Tabagismo } \\
\hline Fumante & $27(93,1)$ & $1,25(0,26-8,16)$ & 1,00 \\
\hline Não fumante & $237(91,5)$ & 1,0 & \\
\hline Ex-fumante & $51(89,5)$ & $0,79(0,28-2,3)$ & 0,62 \\
\hline \multicolumn{4}{|l|}{ IMC (kg/m²) } \\
\hline$<25$ & $113(93,4)$ & 1,0 & \\
\hline$\geq 25$ & $138(93,9)$ & $1,09(0,37-3,19)$ & 0,87 \\
\hline \multicolumn{4}{|l|}{ Saúde de modo geral } \\
\hline Muito bom & $48(96)$ & 1,0 & \\
\hline Bom & $147(89,1)$ & $0,34(0,05-1,61)$ & 0,17 \\
\hline Regular & $97(92,4)$ & $0,51(0,07-2,72)$ & 0,50 \\
\hline Ruim/muito ruim & $23(92)$ & $0,48(0,04-5,17)$ & 0,59 \\
\hline \multicolumn{4}{|l|}{ Plano de Saúde } \\
\hline Sim & $183(92,9)$ & 1,0 & \\
\hline Não & $132(89,2)$ & $0,63(0,28-1,42)$ & 0,22 \\
\hline
\end{tabular}

$\mathrm{OR}=$ Odds Ratio; $\mathrm{IC}=$ Intervalo de confiança. 
Tabela III - Realização de exame de mamografia segundo características sociodemográficas e comportamentos relacionados à saúde. Maringá-PR, 2012.

\begin{tabular}{|c|c|c|c|}
\hline Variáveis & $\begin{array}{c}\text { Realização de } \\
\text { Mamografia } \\
\text { n (\%) } \\
\end{array}$ & $\begin{array}{c}\text { OR } \\
(\text { IC95\%) }\end{array}$ & p valor \\
\hline \multicolumn{4}{|l|}{ Idade } \\
\hline $40-59$ & $122(84,1)$ & 1,0 & \\
\hline$\geq 60$ & $106(86,2)$ & $1,18(0,57-2,45)$ & 0,64 \\
\hline \multicolumn{4}{|l|}{ Escolaridade } \\
\hline $0-8$ & $146(83,9)$ & $0,35(0,05-1,62)$ & 0,18 \\
\hline $9-11$ & $52(83,9)$ & $0,35(0,05-1,87)$ & 0,21 \\
\hline 12 ou mais & $30(93,8)$ & 1,0 & \\
\hline \multicolumn{4}{|l|}{ Estado Civil } \\
\hline Casada & $156(87,2)$ & 1,0 & \\
\hline Solteira & $13(68,4)$ & $0,07(0,01-0,36)$ & $<0,01$ \\
\hline Divorciada/Separada/Viúva & $59(84,3)$ & $0,79(0,34-1,85)$ & 0,55 \\
\hline \multicolumn{4}{|l|}{ Cor } \\
\hline Branca & $153(85,4)$ & 1,0 & \\
\hline Negra & $5(71,4)$ & $0,42(0,07-3,36)$ & 0,28 \\
\hline Parda & $59(85,5)$ & $1,0(0,43-2,38)$ & 0,99 \\
\hline Amarela & $11(84,6)$ & $0,93(0,18-6,49)$ & 1,00 \\
\hline \multicolumn{4}{|l|}{ Ocupação remunerada } \\
\hline Sim & $61(81,9)$ & 1,0 & \\
\hline Não & $167(86,1)$ & $1,32(0,60-2,87)$ & 0,45 \\
\hline \multicolumn{4}{|l|}{ Atividade física no lazer } \\
\hline Ativo & $32(91,4)$ & 1,0 & \\
\hline Inativo & $196(84,1)$ & $2,01(0,55-8,72)$ & 0,25 \\
\hline \multicolumn{4}{|l|}{ Consumo abusivo de álcool } \\
\hline $\operatorname{Sim}$ & $7(70)$ & $0,39(0,09-2,01)$ & 0,17 \\
\hline Não & $221(85,7)$ & 1,0 & \\
\hline \multicolumn{4}{|l|}{ Tabagismo } \\
\hline Fumante & $17(85)$ & $0,98(0,25-4,50)$ & 1,00 \\
\hline Não fumante & $173(85,2)$ & 1,0 & \\
\hline Ex-fumante & $38(84,4)$ & $0,94(0,36-2,55)$ & 0,89 \\
\hline \multicolumn{4}{|l|}{ IMC (kg/m²) } \\
\hline$<25$ & $69(84,14)$ & 1,0 & \\
\hline$\geq 25$ & $108(90)$ & $1,70(0,68-4,25)$ & 0,21 \\
\hline \multicolumn{4}{|l|}{ Saúde de modo geral } \\
\hline Muito bom & $25(83,3)$ & 1,0 & \\
\hline Bom & $101(83,5)$ & $1,01(0,30-3,23)$ & 1,00 \\
\hline Regular & $83(87,4)$ & $1,38(0,38-4,8)$ & 0,55 \\
\hline Ruim/muito ruim & $19(86,4)$ & $1,27(0,22-7,80)$ & 1,00 \\
\hline \multicolumn{4}{|l|}{ Plano de Saúde } \\
\hline $\operatorname{Sim}$ & $145(91,8)$ & 1,0 & \\
\hline Não & $83(75,5)$ & $0,28(0,13-0,59)$ & $<0,01$ \\
\hline
\end{tabular}

IMC=Índice de massa corporal; IC=Intervalo de confiança. 
Tabela IV - Análise multivariada para a realização de exame de Papanicolau de acordo com as variáveis incluídas no modelo. Maringá-PR, 2012.

\begin{tabular}{lccc}
\hline Variáveis & OR ajustada & IC (95\%) & valor $\boldsymbol{p}$ \\
\hline Escolaridade (anos) & & & \\
$\quad 0-8$ & 2,12 & $0,67-6,73$ & 0,19 \\
9-11 & 0,32 & $0,12-0,81$ & 0,01 \\
Estado Civil & & & \\
$\quad$ Solteira & 0,45 & $0,15-1,38$ & 0,16 \\
$\quad$ Divorciada/Separada/Viúva & 1,13 & $0,38-3,40$ & 0,81 \\
Saúde de modo geral & & & \\
$\quad$ Bom & 3,27 & $0,71-15,03$ & 0,12 \\
$\quad$ Regular & 2,24 & $0,44-11,41$ & 0,33 \\
$\quad$ Ruim / muito ruim & 2,55 & $0,32-20,42$ & 0,37 \\
\hline
\end{tabular}

OR=Odds Ratio, IC=Intervalo de confiança.

Tabela V - Análise multivariada para a realização de mamografia de acordo com as variáveis incluídas no modelo. MaringáPR, 2012.

\begin{tabular}{lccc}
\hline Variáveis & OR ajustada & IC (95\%) & valor $\boldsymbol{p}$ \\
\hline Escolaridade & & & \\
$0-8$ & 2,85 & $0,57-14,07$ & 0,19 \\
9-11 & 2,50 & $0,47-13,17$ & 0,27 \\
Estado Civil & & & \\
Solteira & 0,32 & $0,10-1,00$ & 0,05 \\
Divorciada/Separada/Viúva & 0,35 & $0,09-1,27$ & 0,11 \\
Consumo abusivo de álcool & 2,79 & $0,59-13,04$ & 0,19 \\
$\quad$ Não possuir plano de saúde & 0,30 & $0,14-0,62$ & $<0,01$ \\
\hline
\end{tabular}

OR= Odds Ratio; $\mathrm{IC}=$ Intervalo de confiança.

\section{DISCUSSÃO}

Observou-se na população de Maringá que 91,3\% das mulheres com idade superior a 20 anos realizaram exame de Papanicolau, e destas, $84,6 \%$ o realizaram nos últimos três anos. Esse achado demonstra uma triagem eficaz, visto que a $\mathrm{OMS}^{(12)}$ considera suficiente uma cobertura de $80 \%$ do teste de Papanicolau.

Com relação à cobertura do câncer de útero, uma pesquisa realizada em 2011 nas capitais brasileiras verificou que $80,5 \%$ das mulheres entre 25 e 59 anos realizaram citologia oncótica nos últimos três anos. Curitiba e São Paulo apresentaram maior prevalência de realização desse exame, perfazendo $90 \%$ das mulheres avaliadas ${ }^{(13)}$.

Estudo realizado com 404 mulheres no município de São José de Ribamar, Maranhão, identificou que 74,3\% realizaram o exame preventivo em 2004 e, segundo os autores, embora as mulheres tenham conhecimento e realizem o exame, ainda há necessidade de aprimorar a adesão para que as taxas de incidência e mortalidade sejam reduzidas $^{(14)}$. Pesquisa de base populacional com mulheres do município de Maringá apontou uma taxa de $87,6 \%$ de cobertura de Papanicolau na faixa etária de 25-59 anos ${ }^{(15)}$.
Uma revisão de literatura com marco temporal compreendido de 2006 a 2011 aponta os motivos que levam a não realização do exame de forma periódica por algumas mulheres, dentre eles: pouca escolaridade, ausência de companheiro, mulheres mais jovens e também as de idade mais avançada, indisponibilidade de horários, dificuldade de acesso ao serviço de saúde, medo de realizá-lo e/ou de um resultado positivo para o câncer, e constrangimento ${ }^{(16)}$.

No presente estudo, o fato de apresentar escolaridade entre 9 e 11 anos de estudo foi associado à menor prática de Papanicolau. De acordo com a prevalência de realização dos exames conforme características sociodemográficas e comportamentos de saúde ${ }^{(1)}$, encontrou-se menor prevalência de realização do exame de Papanicolau em mulheres com baixa escolaridade (até elementar incompleto).

Pesquisa referente à cobertura de Papanicolau por dois inquéritos domiciliares realizados no município de São Paulo, em 1987 e em 2001-2002, com 968 e 1.125, respectivamente, demonstrou que, do primeiro inquérito para o segundo, houve aumento de $68,8 \%$ para $85,0 \%$ na porcentagem de realização do exame, com as de nível de escolaridade mais baixo demonstrando maiores aumentos nas coberturas ${ }^{(17)}$. 
O nível de escolaridade, tanto baixo quanto alto, é fator importante associado a não realização do teste de Papanicolau. Acredita-se que, pelo avanço das mulheres no mercado de trabalho e a sobrecarga de atividades domésticas, muitas postergam o cuidado com sua saúde, demonstrando que os conceitos de prevenção e promoção ainda se mostram distante da real valorização da manifestação clínica de uma doença $^{(18)}$.

Ao estudar fatores de risco para o câncer de mama entre mulheres de 40 a $69 \operatorname{anos}^{(19)}$, os autores afirmaram existir algumas características sociodemográficas que podem estar associadas ao nível de conhecimento das mulheres sobre os fatores de risco para a doença. Além disso, colocaram que a investigação dessas características pode subsidiar a identificação de fatores que as levam a uma maior participação em ações de detecção precoce.

Com relação à realização de mamografia, a meta de cobertura estabelecida no Healthy People ${ }^{(20)}$ é de $70 \%$ a cada um ou dois anos, em mulheres com 40-69 anos de idade. No presente estudo, essa meta foi alcançada, perfazendo um total de $85,1 \%$ da cobertura em mulheres com idade superior aos 40 anos - destas, 74,3 fizeram a mamografia nos últimos dois anos. Dados recentes apontam que 73,3\% das mulheres entre 50 e 69 anos realizaram mamografia nos últimos dois $\operatorname{anos}^{(9)}$.

O Ministério da Saúde ${ }^{(21)}$ estabelece o parâmetro de um mamógrafo para cada 240 mil habitantes. O Paraná apresenta hoje 159 mamógrafos (1/63 mil habitantes), e a cidade de Maringá dispõe de um número de mamógrafos suficiente para atender à população e assegurar um programa de rastreamento organizado, tanto do setor público quanto do privado.

Um estudo de inquérito populacional realizado com mulheres adultas de 40 a 59 anos e idosas de 60 a 69 anos, residentes em Florianópolis, Santa Catarina, identificou uma prevalência de realização de mamografia anual de $43 \%$ e 38,3\%, respectivamente ${ }^{(22)}$.

A mamografia foi realizada de modo inferior nas mulheres sem plano de saúde privado. Isso pode ser explicado pela falta de rotina médica ao ginecologista, a qual pode ser vista como desnecessária pelas mulheres, por se acharem distantes de problemas advindos da vida sexual ativa e pela falta de motivação e cobertura populacional por parte do serviço público de saúde (agentes comunitários, núcleos de apoio e equipes de saúde da família), o qual deve focar na busca de um melhor acesso e qualidade da atenção básica.

Um estudo longitudinal realizado com 460 mulheres atendidas em serviços de saúde públicos e privados do município de Taubaté, São Paulo, mostrou que 68\% delas referiam rastreamento mamográfico nos dois anos anteriores à pesquisa, sendo maior a proporção $(84,2 \%)$ entre aquelas que tinham cobertura de planos de saúde privados ${ }^{(23)}$. Após análise multivariável, uma pesquisa mostrou como fatores importantes para realização da mamografia o fato de ter plano de saúde, o que indicou maior incorporação e acesso ao exame. Além disso, neste estudo, para as mulheres com idade entre 40 e 69 anos, faixa etária em que o procedimento pode ser mais efetivo, o acesso é mais difícil para quem não tem plano de saúde ${ }^{(18)}$.

Estudo de 27 capitais de estados analisou por inquérito telefônico, em 2008, o monitoramento das doenças crônicas não transmissíveis em 54.353 pessoas com 18 ou mais anos de idade, das quais $41,8 \%$ eram beneficiárias de planos de saúde. Notou-se que a cobertura desses planos proporcionaram aumento na realização de exame mamográfico, sendo identificada uma cobertura de 83,6\% entre aquelas com 50 a 69 anos de idade ${ }^{(24)}$.

$\mathrm{O}$ fato de possuir plano de saúde se mostra relacionado ao melhor poder aquisitivo; além disso, é importante citar que distribuição heterogênea de equipamentos, serviços e recursos humanos são fatores muito importantes no acesso aos serviços ${ }^{(25)}$, quando comparados público e privado.

Vale salientar algumas limitações relevantes neste trabalho. Por se tratar de informações autorreferidas pelas mulheres, podem gerar viés de informação, através de relato com erros por esquecimento ou simulação. Como os dados provêm de inquérito domiciliar cujo questionário utilizado não era elaborado com variáveis relacionadas a fatores de risco para doenças da mama e do útero, dados importantes relacionados às neoplasias não foram acrescentados.

\section{CONCLUSÃO}

A cobertura da mamografia e do Papanicolau foi satisfatória entre as mulheres da cidade de Maringá, Paraná. A baixa escolaridade e as mulheres que dependiam da rede pública de saúde tiveram menor adesão à realização da mamografia.

As reflexões sobre tal situação e suas perspectivas são fundamentais para propor estratégias de promoção da saúde, vigilância, prevenção e atenção.

\section{REFERÊNCIAS}

1. Ministério da Saúde (BR), Instituto Nacional de Câncer - INCA. Coordenação de Prevenção e Vigilância Estimativa 2014: Incidência de Câncer no Brasil. Rio de Janeiro: Ministério da Saúde; 2014.

2. World Health Organization - WHO, International Agency for Research on Cancer. Globocan 2012: estimated câncer incidence, mortality and prevalence worldwide [acesso em 2014 Maio 14]. Disponível em: http://globocan.iarc.fr/Pages/fact_sheets_cancer. aspx. 
3. Silva RCF, Hortale VA. Rastreamento do Câncer de Mama no Brasil: Quem, Como e Por quê? Revista Brasileira de Cancerologia. 2012; 58(1):67-71.

4. Ministério da Saúde (BR), Secretaria de Atenção à Saúde, Secretaria de Atenção à Saúde. Controle dos cânceres do colo do útero e da mama. $2^{\mathrm{a}}$ ed. Brasília: Ministério da Saúde; 2013.

5. Ministério da Saúde (BR), Instituto Nacional de Câncer - INCA. Estimativa 2011: Diretrizes brasileiras para o rastreamento do câncer do colo do útero. Rio de Janeiro: Ministério da Saúde; 2011.

6. Instituto Brasileiro de Geografia e Estatística - IBGE, Ministério do Planejamento, Orçamento e Gestão (BR). Primeiros resultados do Censo 2010. Brasília: IBGE; 2011.

7. Bonita R, Beaglehole R, Kjellstron T. Basic Epidemiology. $2^{\text {nd }}$ edition. Geneva: World Heatlh Organization, 2006.

8. Segri NJ, Priscila MSBF, Maria CGPA, Marilisa BAB, Chester LGC, Moisés G, et al. Práticas preventivas de detecção de câncer em mulheres: comparação das estimativas dos inquéritos de saúde (ISA - Capital) e vigilância de fatores de risco e proteção para doenças crônicas por inquérito telefônico (VIGITEL - São Paulo). Rev Bras Epidemiol. 2011;14(Supl 1):31-43.

9. Ministério da Saúde (BR), Secretaria de Vigilância em Saúde, Secretaria de Gestão Estratégica e Participativa. VIGITEL Brasil 2008: Vigilância de fatores de risco e proteção para doenças crônicas por inquérito telefônico. Brasília: Ministério da Saúde; 2009.

10. World Health Organization - WHO. The World Health Report - Reducing Risks, Promoting Healthy Life. Geneva: WHO; 2004.

11. World Health Organization - WHO. Obesity and overweight. Fact sheet $n^{\circ}$ 311. [acesso em 2014 Maio 14]. Disponível em: http://www.who.int/mediacentre/ factsheets/fs311/en/

12. Ministério da Saúde (BR), Secretaria de Atenção à Saúde. Departamento de Atenção Básica. Controle dos cânceres do colo do útero e da mama. Brasília: Ministério da Saúde; 2006.

13. Ministério da Saúde (BR), VIGITEL Brasil 2011: Vigilância de fatores de risco e proteção para doenças crônicas por inquérito telefônico. Brasília: Ministério da Saúde; 2012.

14. Oliveira AF, Cunha CLF, Viégas IF, Figueiredo IF, Brito LMO, Chein MBC. Estudo sobre a adesão ao exame citopatológico de Papanicolaou em um grupo de mulheres. Rev Pesq Saúde. 2010;11(1):32-7.
15. Murata IMH, Gabrielloni MC, Schirmer J. Cobertura do Papanicolaou em Mulheres de 25 a 59 anos de MaringáPR, Brasil. Rev Bras Cancerol. 2012;58(3):409-15.

16. Silva JMA, Souza RC, Manzo BF, Souza SR, Pereira SM. Fatores relacionados a não continuidade da realização do exame citológico Papanicolaou. Percurso Acadêmico. 2011; 1(2):225-39.

17. Ozawa C, Marcopito LF. Teste de Papanicolaou: cobertura em dois inquéritos domiciliários realizados no município de São Paulo em 1987 e em 2001-2002. Rev Bras Ginecol Obstet. 2011;33(5):238-45.

18. Silva JKS, Santos JA, Silva JS, Amorim ASR. Prevenção do câncer de colo uterino: um enfoque a não adesão. Rev Enferm UFPI. 2013;2(3):53-9.

19. Batiston AP, Tamaki EM, Souza LA, Santos MLM. Conhecimento e prática sobre os fatores de risco para o câncer de mama entre mulheres de 40 a 69 anos. Rev Bras Saúde Matern Infant. 2011;11(2):163-71.

20. Centers for Disease Control and Prevention. Healthy people 2010 [acesso em 2014 Maio 14]. Disponível em: http://www.healthypeople.gov

21. Ministério da Saúde (BR). Portaria $n^{0} 1101$, de 12 de junho de 2002. Estabelece parâmetros assistenciais do SUS. Diário Oficial da União; 2002.

22. Schneider IJC, Giehl MWC, Boing AF, D'orsi E. Rastreamento mamográfico do câncer de mama no Sul do Brasil e fatores associados: estudo de base populacional. Cad Saúde Pública. 2014;30(9):1987-97.

23. Marchi AA, Gurgel MSC. Adesão ao rastreamento mamográfico oportunístico em serviços de saúde públicos e privados. Rev Bras Ginecol Obstet. 2010; 32(4):191-7.

24. Malta DC, Moura EC, Oliveira M, Santos FP. Usuários de planos de saúde: morbidade referida e uso de exames preventivos, por inquérito telefônico, Brasil, 2008. Cad Saúde Pública. 2011;27(1):57-66.

25. Instituto Brasileiro de Geografia e Estatística - IBGE. Pesquisa Nacional por Amostra de Domicílios. Um panorama da saúde no Brasil. Acesso e utilização de serviços, condições de saúde e fatores de risco e proteção à saúde 2008. Rio de Janeiro: IBGE; 2010.

\section{Endereço para correspondência:}

Tiara Cristina Romeiro Lopes

Av. Colombo, 5.790

Bairro: Jd. Universitário

CEP: 87020-900 - Maringá - PR - Brasil

E-mail: tiaracri@gmail.com 